\title{
Multiplexed Detection of Lectins Using Integrated Glycan-coated Microring Resonators
}

\author{
Farshid Ghasemi ${ }^{\mathrm{a}}$, Ehsan Shah Hosseini ${ }^{\mathrm{a}}$, Xuezheng Song ${ }^{\mathrm{b}}$, David S. Gottfried ${ }^{\mathrm{c}}$, Maysamreza Chamanzar ${ }^{\mathrm{a}}$, Mehrsa \\ Raeiszadeh $^{\mathrm{d}}$, Richard D. Cummings ${ }^{\mathrm{b}}$, Ali A. Eftekhar ${ }^{\mathrm{a}}$, Ali Adibi ${ }^{\mathrm{a}, *}$ \\ ${ }^{a}$ Department of Electrical and Computer Engineering, Georgia Institute of Technology, Atlanta, GA, 30324 USA \\ ${ }^{b}$ School of Chemical and Biomolecular Engineering, Georgia Institute of Technology, Atlanta, GA, 30322 USA \\ ${ }^{c}$ Institute for Electronics and Nanotechnology, Georgia Institute of Technology, Atlanta, GA, 30324 USA \\ ${ }^{d}$ Department of Biochemistry, Emory University School of Medicine, Atlanta, GA, 30324 USA
}

\begin{abstract}
We present the systematic design, fabrication, and characterization of a multiplexed label-free lab-on-a-chip biosensor using silicon nitride ( $\mathrm{SiN}$ ) microring resonators. Sensor design is addressed through a systematic approach that enables optimizing the sensor according to the specific noise characteristics of the setup. We find that an optimal $6 \mathrm{~dB}$ undercoupled resonator consumes $40 \%$ less power in our platform to achieve the same limit-of-detection as the conventional designs using critically-coupled resonators that have the maximum light-matter interaction. We lay out an optimization framework that enables the generalization of our method for any type of optical resonator and noise characteristics. The device is fabricated using a CMOS-compatible process, and an efficient swabbing lift-off technique is introduced for the deposition of the protective oxide layer. This technique increases the lift-off quality and yield compared to common lift-off methods based on agitation. The complete sensor system, including microfluidic flow cell and surface functionalization with glycan receptors, is tested for the multiplexed detection of Aleuria Aurantia Lectin (AAL) and Sambucus Nigra Lectin (SNA). Further analysis shows that the sensor limit of detection is $2 \times 10^{-6}$ RIU for bulk refractive index, $1 \mathrm{pg} / \mathrm{mm}^{2}$ for surface-adsorbed mass, and $\sim 10 \mathrm{pM}$ for the glycan/lectins studied here.
\end{abstract}

Keywords: Biosensor, Nanophotonics, Sensor Optimization, Noise, Glycoprotein

\section{Introduction}

Integrated photonic resonators are sensitive, on-chip transducers suitable for various sensing applications (Hunt and Armani. 2010 Fan et al. 2008). Their miniature size allows the realization of large microarray sensors on a single chip that is of interest in many biosensing applications such as the detection of DNA (Rong et al., 2008), microRNA (Qavi and Bailey, 2010), toxins 5 (Ghasemi et al., 2013), blood biomarkers (Washburn et al., 2009), and aptamers (Park et al., 2013). The resonance wavelength of a typical resonance-based integrated photonic sensor changes when the desired analyte binds to its surface, resulting in a change in the transmitted power through an optical waveguide that is coupled to the resonator.

Integrated photonic dielectric resonators have been demonstrated in different material platforms including silicon $(\mathrm{Si})(\mathrm{Claes}$ et al. 2010), silicon nitride (SiN) (Heideman et al., 2012 Lee et al. 2010), indium phosphide (InP) (Ciminelli et al., 2013), 10 and polymers (Chao et al. 2006). Stoichiometric SiN is an appropriate choice as it is compatible with both CMOS fabrication processes and the majority of surface functionalization protocols. In addition, SiN has a relatively small thermo-optic coefficient (TOC) making the device less susceptible to temperature variations. Despite several temperature compensation techniques proposed for integrated photonic resonators (Gylfason et al. 2010|Kirk et al., 2011), the temperature difference between the sensor and the reference resonators is still a source of device-level thermal noise. Small TOC of SiN results in the suppression of this 5 thermal effect by one order of magnitude compared to Si, InP, gallium arsenide, and titania (Della Corte et al., 2000 Gülşen and Naci Inci, 2002). Furthermore, stoichiometric SiN has two important advantages in terms of the system cost. First, it can be deposited using inexpensive processes such as low-pressure chemical vapor deposition (LPCVD). Second, it is transparent to near infrared and visible wavelengths, which enables the use of low-cost light sources and Si photodetectors in the system.

\footnotetext{
* Corresponding author. Tel: 1.404.385.2738

Email address: ali.adibi@gatech.edu (Ali Adibi)
} 
In addition to the material platform, the sensor performance depends on its geometry. A proper figure of merit (FOM) for optimization of a sensor is its limit of detection (LOD) (Chamanzar et al., 2010), defined as the smallest quantity of target analyte that the sensor can reliably detect (Ghasemi et al. 2014). For optimization of LOD in resonance-based sensors, typical design procedures seek to minimize $1 /(\mathrm{Q} . \mathrm{S})$, where $\mathrm{Q}$ is the quality factor of the resonance; and $\mathrm{S}$ is its sensitivity, which is defined as the ratio of the resonance shift to the quantity of the analyte bound to the surface of the resonator (Fard et al., 2014). On the other hand, it is a well established design procedure to maximize the signal-to-noise ratio (SNR), with the "signal" typically defined case the optimization requires a compromise between the SNR and Q of the resonator, a unified approach encompassing both of these factors simultaneously is required. Despite important research efforts on the noise performance of resonance-based sensors (White and Fan, 2008), such a unified approach is missing from the literature. Not only this optimization leads to lowest LOD, it is also crucial for large scale integration of highly multiplexed sensors, where power consumption and heat generation are main limitations.

the power consumption at the same LOD level)

In this article, we demonstrate a new concept for the optimization of power consumption in resonance-based integrated photonic sensors. We argue that to achieve optimal LOD, the resonance lineshape curvature at the resonance wavelength is the single important parameter of the lineshape that should be optimized. This parameter includes the contributions of both the linewidth and the extinction (or equivalently, Q and SNR), and thus shrinks the design space into only one dimension. The waveguide-resonator spacing is then used to tune the coupling strength, which in turn tunes the resonance curvature (i.e., the curvature of transmitted power curve of the bus waveguide in the wavelength domain at the resonance wavelength). On the experimental side, a full biosensor based on the optimized elements is fabricated and functionalized with glycan bioreceptor molecules. Glycans are carbohydrate molecules that specifically recognize toxins and other bio-functional molecules (Smith

40 et al. 2010 Song et al. 2011). The solution of target molecules is delivered through a microfluidic flow cell to reduce the response time and minimize the required sample volume (De Vos et al. 2007). Our results show that our optimized sensor can detect multiple analytes with LOD of $1 \mathrm{pg} / \mathrm{mm}^{2}$ for surface-adsorbed mass, which corresponds to a bulk refractive index LOD of $2 \times 10^{-6}$ RIU (Refractive Index Unit).

In Section 2 we explain device concept, fabrication, and sensor packaging. The optimization of the resonator-waveguide coupling of the sensor is elaborated in Section 3 The sensor is used for label-free, specific, and multiplexed detection of Aleuria Aurantia Lectin and Sambucus Nigra Lectin as described in Section 4 The discussion and conclusions are presented in Sections 5 and 6 .

\section{Materials and Methods}

\subsection{Sensor Device Nanofabrication}

50 The sensor array consists of five SiN microring resonators coupled to a common bus waveguide, as shown in Figure 1 a. The width of the bus waveguide and the microrings is $500 \mathrm{~nm}$ to ensure single-mode operation. The outer radius of each microring is about $8 \mu \mathrm{m}$. Slight offsets in the radii of the microrings result in the spectral offsets of their resonance wavelengths. This offset prevents resonance overlap in the spectral domain to avoid crosstalk problem for multiplexed sensing. It should be noted that the addition of organic bio-receptor layers also shifts the resonances of these microring resonators, depending on the size equidistant in the spectrum.

The device thin film stack is fabricated by thermal oxidation of a standard eight-inch $\mathrm{Si}$ wafer to grow $4 \mu \mathrm{m}$ thermal silicon dioxide $\left(\mathrm{SiO}_{2}\right)$ followed by the deposition of $240 \mathrm{~nm}$ stoichiometric SiN using LPCVD. Thermal oxidation and SiN deposition are performed by Rogue Valley Microsystems (Medford, OR, USA). The device pattern is written into ZEP520A electron-beam inductively coupled plasma etching using $\mathrm{CF}_{4}$ chemistry, leaving no SiN pedestal. Standard ZEP520A spin-coat protocol and a dosage of $220 \mu \mathrm{C} / \mathrm{cm}^{2}$ is used for the EBL. We spin ESPACER 300Z (Showa Denko K.K.; Singapore) on baked ZEP520A to prevent excessive EBL charge-up. The resist is rinsed by de-ionized (DI) water for $1 \mathrm{~min}$, developed for $2 \mathrm{~min}$ in amyl acetate, and then soaked for 30s in isopropyl alcohol (IPA). The residual resist after etching is stripped using Microposit remover 1165 (Shipley). 

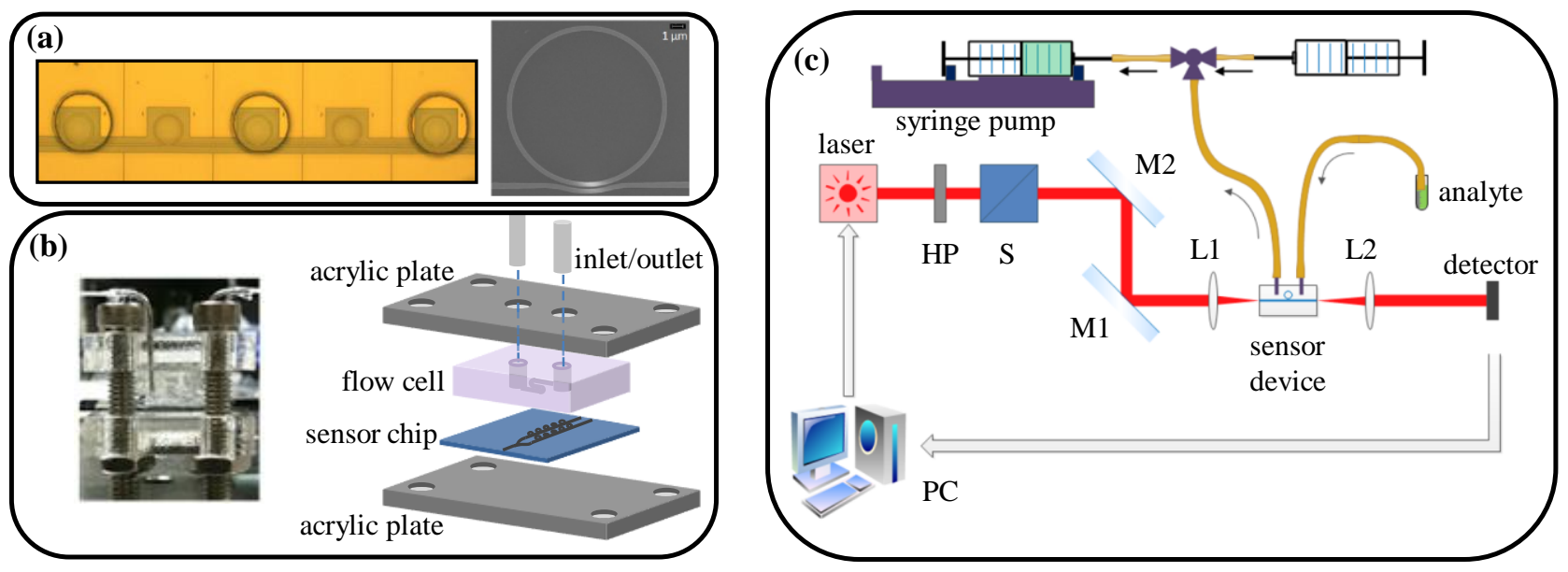

Fig. 1 The components of the sensing system. (a) An optical micrograph of the array of five SiN microrings (left), and a scanning electron micrograph of a SiN microring (right). The width of each microring is $500 \mathrm{~nm}$ to ensure single-mode operation, and its outer radius is about $8 \mu \mathrm{m}$. (b) The sensor chip, PDMS flow cell, and holder structure assembled together. (c) Laser light goes through a half-wave plate (HP), a polarizing beam splitter (S), two alignment mirrors (M1 and M2), and a long-working-distance lens (L1). Using a second long-working-distance lens (L2), the light leaving the chip is projected onto a photodetector, the data of which is sampled by a data acquisition card and processed by a personal computer (PC). A syringe pump in negative pressure mode draws the analyte solution into the PDMS flow cell and then into a waste syringe.

\subsection{Protective Layer: Oxide Lift-off}

Oxide deposition and lift-off are performed to open circular windows above the three sensing microrings on an otherwise oxidecovered chip. To do so, Shipley 1827 resist is spin coated at 500 RPM for $10 \mathrm{~s}$ and then 4000 RPM for 60 s, followed by 1 min bake at $150^{\circ} \mathrm{C}$ on a hotplate. Photolithography is done using $365 \mathrm{~nm}$ UV light with a dosage of $150 \mathrm{~mJ} / \mathrm{cm}^{2}$. Subsequently, the chips are developed in Microposit MF-319 for 50s with gentle agitation, and then rinsed in DI water. A final one-minute descum in oxygen plasma removes the residual resist to promote the adhesion between the SiN film and the oxide layer. As a result, the three sensing microrings are covered by the Shipley 1827 resist while the rest of the substrate is exposed. Using low-temperature plasma-enhanced chemical vapor deposition (PECVD), $2 \mu \mathrm{m}$ of protective oxide is deposited on the chip at a temperature of $100^{\circ} \mathrm{C}$. Next, the chip is submerged in acetone for $10 \mathrm{~min}$ and then gently brushed with an acetone-soaked swab to remove the

75 residue of Shipley 1827 resist and expose the sensing microrings. The chip is finally cleaved to make the waveguides accessible at the edges of the chip for input/output laser coupling. We found the use of a swab more reliable than an ultrasonic bath for oxide lift-off. The force applied by the swab is mainly exerted to the bumps created by the circular patterns of the resist, as opposed to other flat areas. Since the adhesion of low-temperature oxide to the SiN layer is not strong, the ultrasonic agitation can lift the oxide off at undesired areas.

\subsection{Surface Chemistry}

The SiN surface adsorbs biomolecules through various non-specific mechanisms. These mechanisms make the sensor respond to the presence of undesired molecules. To increase the sensor specificity, we covalently immobilize bio-receptor molecules on the surface of the sensor. In the first step, the - $\mathrm{OH}$ bonds of the surface of the chip are activated by cleaning it with piranha (5:1 mix of $\mathrm{H}_{2} \mathrm{SO}_{4}$ and $\mathrm{H}_{2} \mathrm{O}_{2}$ ) for $30 \mathrm{~min}$, a DI water rinse, and a $10 \mathrm{~min}$ exposure to UV/ozone plasma in a UVOCS ${ }^{\circledR}$ cleaner.

The chips are then immersed in a $2 \% \mathrm{v} / \mathrm{v}$ solution of (3-mercaptopropyl)trimethoxysilane in anhydrous toluene for one hour (in a nitrogen ambient), thoroughly rinsed with IPA, dried, and baked at $80^{\circ} \mathrm{C}$ for $20 \mathrm{~min}$. When the samples cool down, a drop of 2 mM SM(PEG) 12 linker (Pierce; Chicago, IL, USA) in anhydrous dimethyl sulfoxide (DMSO, from Acros Organics; Pittsburgh, PA, USA) is placed on the chip to cover its surface. The sample is left unperturbed in a nitrogen ambient for about $12 \mathrm{~h}$, and then thoroughly rinsed with IPA. SM(PEG)12 linker contains a polyethylene glycol (PEG) chain that helps reduce the nonspecific binding of undesired molecules to the surface (De Vos et al. 2009). At this stage, carboxyl functional groups containing $\mathrm{N}$-Hydroxysuccinimide (NHS) esters are covalently grafted on the surface and can immobilize any molecules containing amine 
(a)

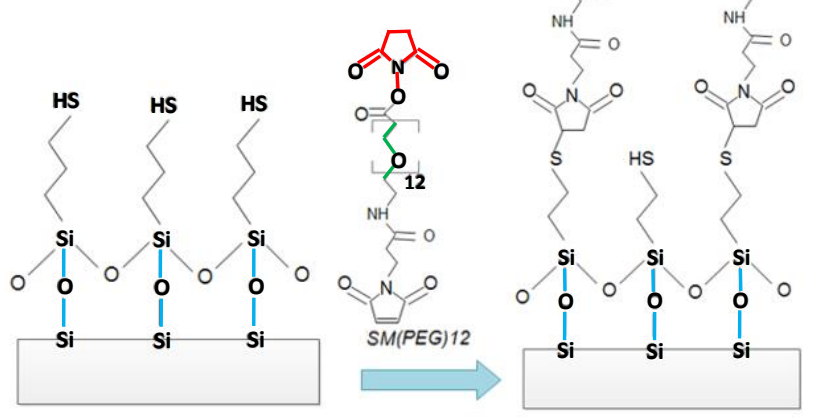

(b)

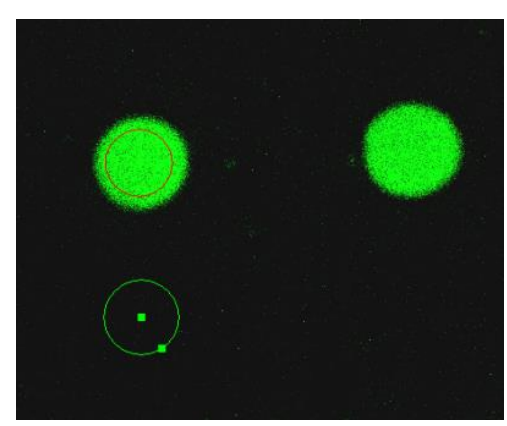

Fig. 2 (a) Schematic representation of the surface chemistry for NHS activation on the SiN surface. Blue: siloxane functional group; red: NHS ester; green: PEG. (b) A fluorescence microscope image showing selective binding of CTB to GM1 glycan with negligible binding to the areas lacking GM1 (but having a PEG brush). The average fluorescence signal intensity is about two orders of magnitude larger in the areas containing GM1 receptors than otherwise.

functional groups. Figure 2 a shows a schematic representation of the NHS activation protocol. All chemicals, except SM(PEG) 12 and DMSO, were obtained from Sigma-Aldrich (St. Louis, MO, US).

A $200 \mathrm{~mm}$ solution of amine-derivatized glycan (Song et al. 2009) is printed selectively on the microrings using a BioForce Nano eNabler. Using this tool, each microring can be coated with a specific glycan. Prior to the printing, the specific glycan solution is mixed 1:1 with phosphate buffered saline (PBS, $\mathrm{pH}$ 7.4) containing $10 \%$ glycerol to impede evaporation of the glycan solution in the open-top reservoir of the printing cantilever. After the glycans are printed, the chip is left at a relative humidity of $80 \%$ to prevent evaporation of the printed droplets. This allows the glycan molecules to bind to the surface through their amine functional groups. Next, the sample is incubated in a high humidity (at about saturation) chamber at $50^{\circ} \mathrm{C}$ for $1 \mathrm{~h}$. The chips are dried and thoroughly washed in a PBS solution with $0.05 \%$ Tween 20, and then in DI water. Remaining NHS esters are de-activated in a solution of $50 \mathrm{~mm}$ ethanolamine in $0.1 \mathrm{M}$ Tris buffer $(\mathrm{pH} \mathrm{9.0)} \mathrm{for} 1 \mathrm{~h}$. This finalizes the glycan immobilization step and prepares the chip for microfluidic integration.

To verify the efficacy and specificity of the activated surface, we tested the binding of the glycans using fluorescence microscopy. GM1 glycan (von Gunten et al. 2009) was immobilized using the above mentioned protocol on the SiN surface, and a $50 \mu \mathrm{g} / \mathrm{ml}$ drop of biotin conjugated Cholera toxin B subunit (CTB from Sigma Aldrich; St. Louis, MO, USA) was placed on the surface for $1 \mathrm{~h}$. The SiN surface was then thoroughly washed with PBS solution containing $0.05 \%$ Tween 20, and then by DI water. Subsequently, a $200 \mu \mathrm{g} / \mathrm{ml}$ drop of Alexa 488 conjugated Streptavidin (Life Technologies; Carlsbad, CA, USA) was placed on the surface for $1 \mathrm{~h}$, followed by same wash process mentioned above. Figure $2 \mathrm{~b}$ shows an image of the surface using a Carl-Zeiss LSM 710 confocal fluorescence microscope. The bright spots in this figure correspond to the areas where the glycans 
have been printed. In these areas, the glycans capture biotin-CTB, and then biotin-CTB captures Alexa-Streptavidin. In other areas, biotin-CTB and thus, Alexa-Streptavidin can not effectively bind to the PEG-coated surface. The image shows a high contrast between the glycan-coated areas and the rest of the surface, which demonstrates a low non-specific binding on the areas lacking glycan receptors.

\subsection{Microfluidic Integration}

115 After glycan immobilization, a polydimethylsiloxane (PDMS) microfluidic flow cell is aligned to the SiN sensor chip using Finetech flip-chip bonder. The microfluidic flow cell helps reduce the response time of the sensor by overcoming the diffusion barrier. As illustrated in Figure 1p, two acrylic plates hold the chip and the flow cell together by applying a gentle force exerted by four cap screws and nuts at the corners. The acrylic plates are cut using a Hermes lasercutter machine. After assembling the structure, inlet and outlet needles are inserted into the holes made by a puncher in the PDMS flow cell. A syringe pump in negative pressure mode draws the analyte, with a flow rate of $2 \mu \mathrm{L} / \mathrm{min}$ into the input tube, the microfluidic chip, and finally the output tube. The microfluidic channel is $100 \mu \mathrm{m}$ wide and $50 \mu \mathrm{m}$ high, and it is cast using an SU-8 mold.

To make the mold, SU-8 2050 (MicroChem; Newton, MA, USA) is spin coated on a Si wafer (500 RPM for 10s, then 2000 RPM for $60 \mathrm{~s}$ ), and baked at $95^{\circ} \mathrm{C}$ for $5 \mathrm{~min}$. The pattern of the microfluidic channel is written into the resist using a Microtech LW405 laserwriter. The sample is then baked, first at $65^{\circ} \mathrm{C}$ for $1 \mathrm{~min}$, and then at $95^{\circ} \mathrm{C}$ for $4 \mathrm{~min}$. Unexposed resist is removed IPA, and further baked at $250^{\circ} \mathrm{C}$ for $30 \mathrm{~min}$. This finalizes the preparation of the mold.

To cast PDMS, Dow Corning Sylgard 184 PDMS base and curing agent (Ellsworth; Loganville, GA, USA) are mixed 10:1 $(\mathrm{w} / \mathrm{w})$, stirred well, and degassed in vacuum for about one hour. The mixture is poured into the mold, degassed in vacuum for about one hour, and baked at $90^{\circ} \mathrm{C}$ for about 10 hours to produce the PDMS microfluidic flow cell.

\subsection{Biosensor Characterization Setup}

As shown in Figure 1F, light from a tunable laser $(652-660 \mathrm{~nm}$, Newport TLB 6305) is passed through a half-wave plate, a polarizing beam splitter, and a long-working-distance lens to have it focused at the input facet of the sensor chip. Dominant polarization axis of the laser light is rotated by the half-wave plate (HP) to make its magnetic field parallel to the surface of the sensor chip (TM polarization). The light exiting the output waveguide is projected onto a photodetector (Thorlabs PDA36A silicon amplified detector) using another long working distance lens. The laser is controlled by LabVIEW software that scans the wavelength over the $652.4-660 \mathrm{~nm}$ window, while the readout of the photodecetor is synchronously recorded by a USB6211 National Instrument data acquisition (DAQ) device. The readout is de-noised in LabView software using a third order Butterworth low-pass filter with a cut-off frequency of $100 \mathrm{~Hz}$. The laser scan rate is $1 \mathrm{~nm} / \mathrm{s}$, and sampling resolution is $0.25 \mathrm{pm}$. Normalized transmission spectrum is calculated by dividing the transmitted power to the baseline power. The baseline power is calculated by low-pass filtering the transmitted power to remove higher frequency features of the spectrum. An in-house data analysis software in MATLAB environment tracks the resonance shifts in time.

\section{Theory: Optimization}

\subsection{Figure of Merit}

Waveguide-resonator coupling, as seen in Figure $1 \mathrm{a}$, determines the extinction and the loaded Q of the resonance. The strength of this coupling depends on the gap between the waveguide and the resonator. Proper choice of this gap helps maximize resonance sharpness. A sharper lineshape helps detect the resonance more accurately at any specific noise level (Ghasemi et al., 2015).

An important aspect of the resonance detection procedure is the data processing algorithm employed to extract the resonance wavelength from the experimental data. In this paper, we use a quadratic fit to the data around the resonance as the resonance detection algorithm. A quadratic fit using linear regression is a fast, universal, and versatile method that can rival more sophisticated but sensitive detection methods such as a nonlinear parametric fit (Gylfason et al. 2010). Although parametric fits in general are more accurate when we have an accurate parametric model of the lineshape (e.g., Lorentzian function), in practice the uncertainty about the actual resonance function limits the performance of these parametric fits. The discrepancy between the model and actual lineshape comes from various sources of reflection in the device, such as fabrication imperfections, and the edges of circular openings on the waveguides, which result in lineshape deformation. In this condition, a Lorentzian fit does not 

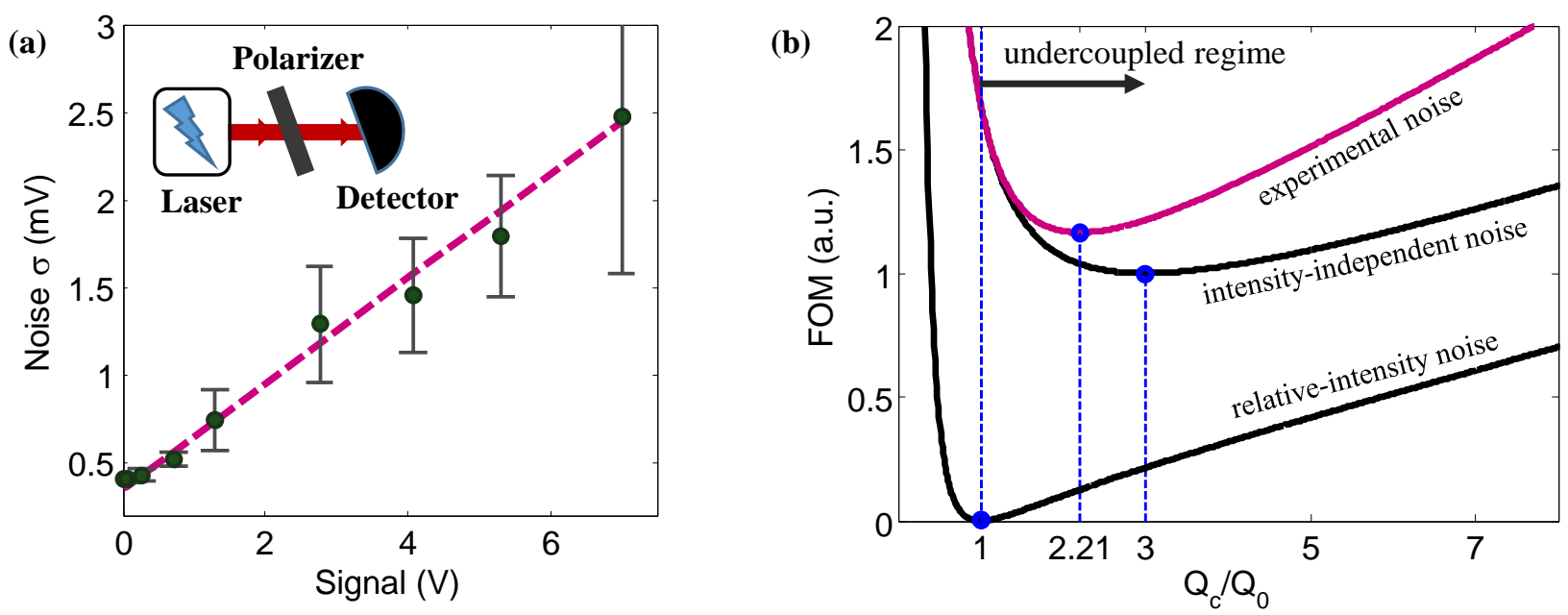

Fig. 3 (a) Measured noise intensity as the average intensity of incoming light on the photodetector changes. Since the laser output is partially polarized, a polarizer serves as a variable attenuator to control the average intensity of the light on the photodetector. (b) The FOM

(Equation 5) for different ratios of coupling Q $\left(Q_{c}\right)$ and the intrinsic $\mathrm{Q}\left(Q_{0}\right)$. The FOM corresponding to the experimentally measured noise, is compared with the theoretical cases for intensity-independent noise $\left(\sigma_{n}=0.35 \mathrm{mV}\right)$ and relative-intensity noise $\left(\sigma_{n}=0.3 V(\mathrm{mV})\right)$. The circles show the optimal FOM on each curve.

necessarily lead to more accurate detection of the resonance wavelength. In contrast, for the majority of mechanisms leading to a lineshape deformation, the lineshape generally follows a quadratic function around the resonance wavelength.

The Lorentzian lineshape of a microring resonance can be approximated by a quadratic polynomial around the center of the resonance lineshape. The quadratic polynomial is determined by two parameters: its curvature $\left(\kappa_{0}\right)$, and the amplitude of its minimum point (i.e., a vertical offset). The latter does not affect the resonance detection accuracy because a homogeneous shift in the amplitudes of all data points does not shift the resonance along the wavelength axis. Thus, as far as detection accuracy is concerned, the only important parameter is the lineshape curvature.

Beside the lineshape curvature, noise level should also be considered in the coupling optimization. If the noise is intensityindependent, the extinction of the resonance does not affect the noise level. However, for an intensity-dependent noise, the extinction determines the noise level around the resonance dip. It is therefore necessary for the optimization procedure to simultaneously minimize the noise level $\left(\sigma_{n}\right)$ while maximizing the curvature $\left(\kappa_{0}\right)$. The end goal of this optimization is to minimize the error in resonance detection when the measurement contains noise. In this regard, it should be noted that an identical scaling of all measurements (i.e., the lineshape and the noise level) does not change the resonance detection accuracy. Hence, we would like the FOM not to change under such a scaling. Hence, we define the FOM as the ratio of $\sigma_{n}$ and $\kappa_{0}$, since both parameters scale identically and the scaling factor is eliminated from their ratio:

$$
\mathrm{FOM}=\frac{\sigma_{n}}{\kappa_{0}} .
$$

Although we introduced the FOM in Equation (11) through a discussion of its desired requirements, a rigorous analysis of our resonance detection method (using a quadratic fit) supports this definition for FOM (Ghasemi et al., 2015). By using the FOM defined in Equation (1), the SNR need not be optimized separately from the Q. This FOM is the ultimate criteria for optimizing the performance of the sensor in terms of the amplitude noise, which is the objective of the optimization of Q and SNR.

\subsection{Noise Measurement for Calculation of FOM}

To calculate the FOM in Equation (1), we first study the $\sigma_{n}$ in the numerator. To measure the intensity dependence of noise in our setup, we replace the sensor chip with a polarizer. The polarizer acts as a variable attenuator since the laser light has a major axis of polarization. At any specific wavelength, the sensor device is a linear system with a determined attenuation (and a phase response that is not picked up by the photodetector). Thus, an attenuator can be used to measure the effective noise on every data point of the spectrum. 
For noise measurement, the laser wavelength is scanned from $652.4 \mathrm{~nm}$ to $660 \mathrm{~nm}$ with a constant current of $43.5 \mathrm{~mA}$. Photodetector gain is set to $40 \mathrm{~dB}$. Since laser output power varies slowly over the scan window, the baseline is calculated by applying a 21-point moving average filter to the data in MATLAB environment. The difference between the raw readout and the baseline is considered as the noise. For each specific attenuation (i.e., polarizer angle), the standard deviation of noise $\left(\sigma_{n}\right)$ is calculated over the data in the $654-659 \mathrm{~nm}$ window. The resulting curve in Figure 3 a shows the intensity-dependence of noise as

$$
\sigma_{n}=0.35+0.3 \mathrm{~V}(\mathrm{mV}),
$$

where $V$ is the average light intensity on the photodetector, expressed in terms of the equivalent voltage that the photodetector generates at its output (including the amplification, in Volts).

near-zero signals $(V \sim 0)$ matters in this optimization

On the other hand, the resonance curvature $\left(\kappa_{0}\right)$ and its extinction can be calculated using coupled-mode theory (CMT) (Soltani et al. 2010). The power transmission through a device with a single microring, $T(\lambda)$, is (Shah Hosseini et al. 2010)

$$
T(\lambda)=\frac{P_{\text {out }}}{P_{\text {in }}}=\left|\frac{-2 j\left(\lambda-\lambda_{0}\right) / \lambda_{0}+1 / Q_{0}-1 / Q_{c}}{-2 j\left(\lambda-\lambda_{0}\right) / \lambda_{0}+1 / Q_{0}+1 / Q_{c}}\right|^{2} .
$$

Here, $\lambda_{0}$ and $Q_{0}$ are the resonance wavelength and the intrinsic $\mathrm{Q}$ of the microring, respectively. The observation wavelength is denoted by $\lambda$, and the coupling quality factor by $Q_{c}$. The curvature of this lineshape at its resonance is

$$
\kappa_{0}=\left.V_{0} \frac{\partial^{2} T}{\partial \lambda^{2}}\right|_{\lambda=\lambda_{0}}=32 V_{0}\left(\frac{Q_{0}}{\lambda_{0}}\right)^{2} \frac{\left(Q_{c} / Q_{0}\right)^{3}}{\left(1+Q_{c} / Q_{0}\right)^{4}},
$$

in which $V_{0}$ denotes the photodetector voltage readout for an all-pass transmission.

The FOM can be calculated using the noise characteristics from Equations (2) and (3) and resonance curvature from Equation (47. Assuming a typical all-pass voltage of $V_{0}=1 \mathrm{~V}$ through the device and arbitrary but constant $\lambda_{0}$ and $Q_{0}$, our optimization goal is to minimize Equation (1):

$$
\mathrm{FOM}=\frac{\sigma_{n}\left(V_{0} T\left(\lambda_{0}\right)\right)}{\kappa_{0}} .
$$

We use experimental noise measurements, along with Equation (4) for $\kappa_{0}$ to calculate the FOM. The result is plotted in Figure 3b for different values of $Q_{c} / Q_{0}$. Optimum coupling occurs for $Q_{c} / Q_{0} \approx 2.21$, which corresponds to an extinction of about $8.5 \mathrm{~dB}$ (undercoupled).

\subsection{Optimal Coupling Condition}

It is a widely invoked optimization rationale to choose sensor parameters so that the light-matter interaction is maximized. This condition is achieved at the critical coupling regime, where $Q_{c} / Q_{0}=1$ (Chao et al. 2006). However, as it is seen in Figure 33, the FOM at its optimal point is about 0.69 times that at the critical coupling regime. This means, the LOD improves by $31 \%$ if the optimal coupling, where $Q_{c} / Q_{0}=2.21$, is used rather than critical coupling. The reason is that using a quadratic fit for resonance detection, the $3 \sigma_{\lambda}$ error in resonance detection linearly depends on the FOM defined here; and LOD, in turn, depends linearly on the $3 \sigma_{\lambda}$ error (Ghasemi et al. 2015). In inferring this conclusion for the LOD improvement, it is assumed that the designs are compared for a similar input power.

Alternatively, the improvement in the FOM is equivalent to power consumption reduction at a fixed LOD. An example of a condition at which the optical power is adjusted to meet a determined LOD is when the device performance is limited by noises other than additive amplitude noise (e.g., biochemical noise or wavelength noise). In this case, it is desired to set the power so that the contribution of the amplitude noise to the total $3 \sigma_{\lambda}$ is about the same as that from the other dominant sources of noise (Ghasemi et al., 2014). Any further increase of the power will be mostly wasted as the device is already limited by the other noise mechanisms. In this sense, a critically coupled resonator will require $31 \%$ more power to achieve the same performance as the optimized device discussed above. 

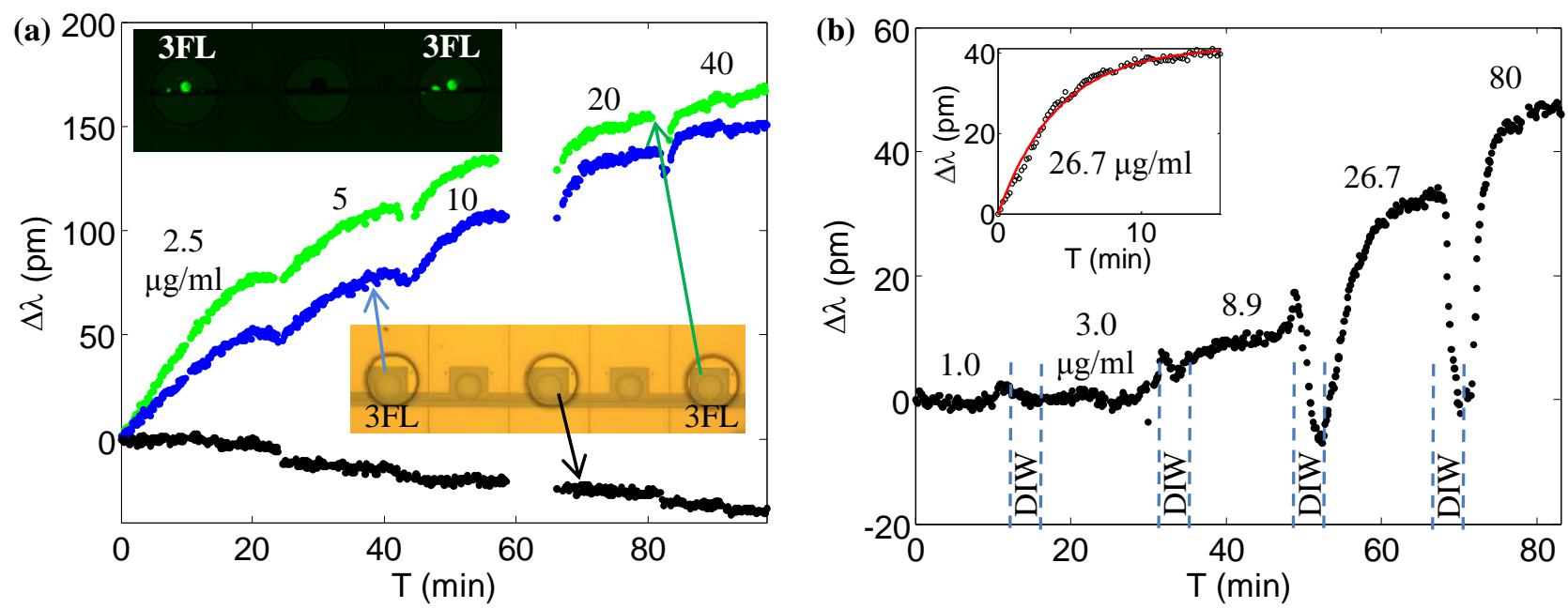

Fig. 4 The individual detection of AAL and SNA. Two separate sensor chips were prepared with their first and last microrings coated with the corresponding glycans, and the middle resonator had a PEG coating without any glycans. The concentration of the lectin in each test cycle is noted on the graph in $\mu \mathrm{g} / \mathrm{ml}$. The resonance shifts of the three sensing resonators are referenced to the average of the resonance shifts of the two oxide-covered resonators. (a) The binding of AAL to 3FL on the surface of the microrings. The inset shows a fluorescence microscope image of the dyed chip. (b) The average of resonance shifts of the two 2,6-NA2 coated resonators referenced to the resonance shift of the PEG-coated (middle) resonator. The inset shows the test cycle corresponding to an SNA concentration of $26.7 \mu \mathrm{g} / \mathrm{ml}$.

\section{Results}

\subsection{Saltwater Titration}

We performed an initial round of fabrication with varying waveguide-resonator gaps to find the gap resulting in an undercoupled $8.5 \mathrm{~dB}$ extinction. Next, we conducted saltwater titration to measure the bulk sensitivity of the resonators. Bulk sensitivity is defined as the resonance shift normalized to the refractive index change in the cladding material. The sensor was tested with different concentrations of saltwater and the resonance shifts were measured eight times for each concentration (data not shown here). Having the refractive index of saltwater (Quan and Fry, 1995) for different concentrations at $25^{\circ} \mathrm{C}$, the experimental bulk sensitivity is calculated to be $49 \mathrm{~nm} / \mathrm{RIU}$ (RIU is the refractive index unit of the clad). The numerical simulation of the microrings in the COMSOL environment results in a bulk sensitivity of $48.1 \mathrm{~nm} / \mathrm{RIU}$. In this calculation, refractive indices of $\mathrm{SiN} \mathrm{SiO}_{2}$, and water clad were assumed to be $2.05,1.44$, and 1.33 , respectively.

\subsection{Lectin Detection: Individual Tests}

We immobilized amine-derivatized (Song et al. 2009) 3-fucosyl lactose (3FL) on two of the exposed microrings for specific detection of biotinylated Aleuria Aurantia Lectin (AAL). All the glycans and lectins in this work were purchased from Vector Labs (Burlingame, CA, USA). The glycan solution was not printed on the middle microring so that this microring could serve as a control for non-specific binding. Different concentrations of AAL were flowed over the sensor using the microfluidics, and the device spectrum was measured every $10 \mathrm{~s}$. The introduction of each solution, as shown in Figure $4 \mathrm{a}$, results in a red shift in the resonance wavelengths of the two glycan-coated microrings. The difference in the shifts of the two microrings can be attributed to the difference in the surface coverage of glycans and sensitivities of the two microrings. The response of the middle microring sensor (lacking any glycan receptors but having a PEG coating) slowly shifts toward shorter wavelengths. This shift can be due to non-covalently bound linker molecules that gradually leave the surface when the analyte flows in the channel. The glycan/lectin dose-response curve is generally expected to follow a Sigmoid curve:

$$
R=R_{s} \frac{C}{C+K_{D}},
$$


Table 1 Resonance shifts and calculated lectin concentrations from the multiplexed test.

\begin{tabular}{ccccc}
\hline \hline & $R_{H}(\mathrm{pm})$ & $R_{s}(\mathrm{pm})$ & $R_{L}(\mathrm{pm})$ & $C_{L}(\mu \mathrm{g} / \mathrm{ml})$ \\
\hline \multirow{2}{*}{ AAL } & 968 & 1104 & 385 & 3.2 \\
SNA & 167 & 219 & 79 & 16 \\
\hline
\end{tabular}

\section{Discussions}

\subsection{Limit of Detection}

With a repeatability of $3 \sigma \sim 0.1 \mathrm{pm}$ for the resonance detection in our system (Ghasemi et al., 2014), and a sensitivity of 49 $\mathrm{nm} / \mathrm{RIU}$ from Section 4.1 the sensor LOD is $2 \times 10^{-6}$ RIU for bulk refractive index sensing. The sensitivity of the microring resonance wavelength to the deposition of an organic monolayer with a refractive index of 1.45 in water is about $109 \mathrm{pm} / \mathrm{nm}$ (resonance shift per homogeneous layer thickness, from COMSOL simulations). This leads to a minimum resolvable thickness of $1 \mathrm{pm}$ that corresponds to an LOD of $1 \mathrm{pg} / \mathrm{mm}^{2}$, which is in the same range as comparable integrated photonic sensor systems based on Si microrings (Luchansky et al., 2010), folded cavities (Xu et al., 2008), and liquid core optical ring resonators (LCORR) (Zhu et al., 2007). 


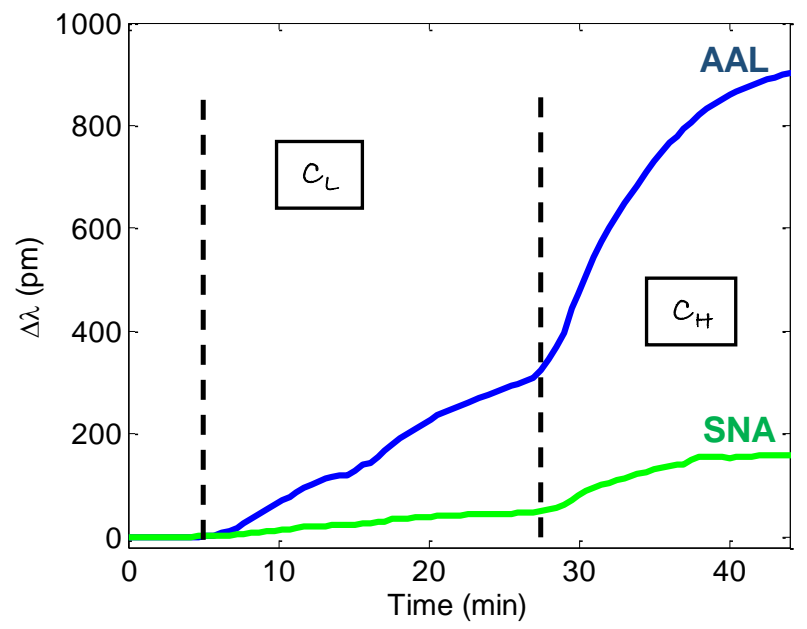

Fig. 5 Sensor response to the mixture of the two lectins (AAL and SNA). The low concentration solution is flowed to the sensor in the $C_{L}$ phase, followed by the high concentration solution in the $C_{H}$ phase. The resonance shift and saturation time constant for each microring depends on the concentration of the corresponding lectin and the binding dynamics of the glycan/lectin pair.

For the detection of lectins, the sensor LOD depends linearly on the saturated response, and thus on the density of immobilized receptors on the surface. According to Equation (7), the smallest concentration corresponding to a minimum resonance shift of $3 \sigma_{\lambda}$ is

$$
L O D \approx K_{D} \frac{3 \sigma_{\lambda}}{R_{s}},
$$

assuming $R_{S} \gg 3 \sigma_{\lambda}$. Hence, according to the saturated resonance shifts $\left(R_{S}\right)$ in Table 1 , the LOD is about $0.5 \mathrm{ng} / \mathrm{ml}$ (or $7 \mathrm{pM}$ ) for AAL detection, and about $12 \mathrm{ng} / \mathrm{ml}$ (or $86 \mathrm{pM}$ ) for SNA detection. The molecular weights of AAL and SNA are $72 \mathrm{kDa}$ and $140 \mathrm{kDa}$, respectively. In the calculation of this LOD only the effects of optical, electrical, and thermal noises were included. The differences between the calculated $C_{L}$ concentrations in Table 1 and the actual concentrations can originate from chip-to-chip variations in the surface chemistry, and the non-uniformity of flow originating from air bubbles in the flow cell. In complex samples, the operating parameters of the sensor may also depend on the presence of interfering species.

\subsection{Coupling Optimization: Special Cases}

We optimized the waveguide-resonator coupling in Section 3 for the specific noise we measured in our setup. In this section, we discuss two alternative, common noise models: 1) intensity-independent noise, and 2) relative-intensity noise. Figure $3 \mathrm{p}$ includes the plots of the FOM for these noise models. It is worth noting that for an intensity-independent noise, a $6 \mathrm{~dB}$ undercoupled condition $\left(Q_{c} / Q_{0}=3\right)$ leads to the optimal FOM. Since the noise is independent of the intensity, our optimization approach seeks the coupling condition that results in the sharpest resonance. Although at critical coupling the extinction is maximum, the loaded resonance curvature is smaller than the $6 \mathrm{~dB}$ undercoupled case. According to our optimization procedure, the $6 \mathrm{~dB}$ undercoupled condition is the ideal compromise between the extinction and the linewidth. At this coupling strength, the FOM is $40 \%$ smaller than that for the critically-coupled case, which results in $40 \%$ improvement in the LOD (at a fixed optical power), or $40 \%$ less power consumption (for a fixed LOD).

For a relative-intensity noise, on the other hand, the optimal condition is critical coupling. In this case, the noise vanishes around the resonance wavelength of a critically-coupled resonator. That is because the output light intensity vanishes at the resonance, and so does the relative-intensity noise by its definition. Hence, the critical coupling regime results in a nearly zero noise level around the resonance and it is thus, the optimal design. In practice, however, an ideal relative-intensity noise that totally vanishes at the zero signal level is rare. Multiple sources of amplitude noise contributing to the aggregate amplitude noise are independent of the light reaching the detector (for instance, detector dark noise, electronic noise, etc.), and they result in residual noise. 


\subsection{Oxide Deposition Temperature for Proper Lift-off}

We used low-temperature $\left(100^{\circ} \mathrm{C}\right)$ oxide for the deposition of the protective oxide layer. Although the deposition at higher temperatures (e.g., around $250^{\circ} \mathrm{C}$ ) results in a higher quality PECVD oxide, such high temperatures change the chemical characteristics of the resist, and the resist cannot be developed using regular developer solutions after oxide deposition. For this reason, we used low-temperature oxide deposited by an Oxford ICP PECVD tool. We also tried oxide deposition using a CHA Industries electron-beam evaporator. This method of deposition does not change the chemical characteristics of Shipley 1827 resist, but the deposition takes longer, requires periodic monitoring, and more variations were observed in the quality of the deposited layer from one fabrication round to the next.

\subsection{Bioreceptor Density on Sensor Surface}

We used BioForce Nano eNabler tool to print 3FL glycan first, and then 2,6-NA2, as explained in Section 2.3 . The printing process requires a high humidity (a relative humidity of about $80 \%$ in our case) for the analyte to flow from the reservoir to the surface. This high humidity deactivates water-sensitive NHS functional groups. Therefore, the later the glycan solution is printed, the fewer NHS groups will be available for covalent immobilization of the glycan molecules. This process results in a relatively lower level of response for SNA compared to AAL in Figure 5. This trend was observed in other similarly prepared sensor chips as well.

\section{Conclusion}

Total system cost is one of the commercialization challenges for highly multiplexed biosensors based on integrated photonic resonators. In this article a label-free opto-fluidic sensor was presented that benefits from a low-cost silicon nitride device layer and silicon photodetectors. Conventional design rules of thumb recommend maximum light-matter interaction, which occurs 315 at critical coupling for traveling-wave resonators. However, we showed that while the critical coupling condition is optimum in the presence of a relative-intensity noise, the optimum design in the presence of an ideal intensity-independent noise is an undercoupled resonator with a 6-dB extinction. The latter design has a 40\% power (or equivalently, LOD) advantage over conventional critically-coupled designs. For the specific noise characteristics measured in our setup, the optimal design consumes about $31 \%$ less power than a critically-coupled configuration for a fixed LOD. Multiplexed label-free detection experiment was performed for Aleuria Aurantia Lectin (AAL) and Sambucus Nigra Lectin (SNA). 3FL and 2,6-NA2 glycans were used as specific bio-receptors for the detection of these lectins. Further analysis shows that the sensor limit of detection is $\sim 2 \times 10^{-6}$ RIU for bulk refractive index, $\sim 1 \mathrm{pg} / \mathrm{mm}^{2}$ for surface-adsorbed mass, and $\sim 10 \mathrm{pM}$ for the glycan/lectin pairs studied here. The performance of our sensor is comparable to the state of the art, however with the critical advantages of lower power consumption, relative structural simplicity, and low cost. The experimental and theoretical study in this paper paves the way toward the realization of

highly-multiplexed and dense micro-array sensors.

\section{Contributions}

FG, AAE, and AA planned the project. FG, ESH, and MC fabricated the photonic chip; FG, DSG, AAE, MC, ESH, and MR developed the surface chemistry and fluidic packaging. XS and RDC supplied the material and protocol for glycan immobilization. FG and MC characterized the sensor. FG, AAE, and DSG analyzed the data. Noise performance optimization was carried out by

FG and AA. All authors edited and commented on the manuscript.

\section{Acknowledgment}

This work was performed in part at the Georgia Tech Institute for Electronics and Nanotechnology (IEN), a member of the National Nanotechnology Infrastructure Network, which is supported by the National Science Foundation. The authors gratefully acknowledge the staff of the IEN, especially Devin Brown. The authors thank following people for helpful discussions: Hamed Shams-Mousavi on surface chemistry; Amir Hossein Atabaki and Siva Yegnanarayanan on optical characterization; Reza Abbaspour on microfluidics fabrication; Andrew Shaw on fluorescence microscopy; and Mahmoud Mahmoud and Seth Marder on surface chemistry. This work was supported by the Defense Advanced Research Projects Agency (DARPA) under Contract No. HR 0011-10-1-0075. 


\section{References}

${ }_{340} 1$ Chamanzar, M., Soltani, M., Momeni, B., Yegnanarayanan, S., Adibi, A., 2010. Hybrid photonic surface-plasmon-polariton ring resonators for sensing applications. Applied Physics B 101 (1-2), 263-271.

2 Chao, C.-Y., Fung, W., Guo, L. J., 2006. Polymer microring resonators for biochemical sensing applications. Selected Topics in Quantum Electronics, IEEE Journal of 12 (1), 134-142.

3 Ciminelli, C., Dell'Olio, F., Armenise, M. N., Soares, F. M., Passenberg, W., 2013. High performance inp ring resonator for new 345 generation monolithically integrated optical gyroscopes. Optics express 21 (1), 556-564.

4 Claes, T., Bogaerts, W., Bienstman, P., 2010. Experimental characterization of a silicon photonic biosensor consisting of two cascaded ring resonators based on the vernier-effect and introduction of a curve fitting method for an improved detection limit. Optics express 18 (22), 22747-22761.

5 De Vos, K., Bartolozzi, I., Schacht, E., Bienstman, P., Baets, R., 2007. Silicon-on-insulator microring resonator for sensitive and label-free biosensing. Optics express 15 (12), 7610-7615.

6 De Vos, K., Girones, J., Popelka, S., Schacht, E., Baets, R., Bienstman, P., 2009. Soi optical microring resonator with poly (ethylene glycol) polymer brush for label-free biosensor applications. Biosensors and Bioelectronics 24 (8), 2528-2533.

7 Della Corte, F. G., Cocorullo, G., Iodice, M., Rendina, I., 2000. Temperature dependence of the thermo-optic coefficient of inp, gaas, and sic from room temperature to $600 \mathrm{k}$ at the wavelength of $1.5 \mu \mathrm{m}$. Applied physics letters 77 (11), 1614-1616.

${ }_{355} 8$ Fan, X., White, I. M., Shopova, S. I., Zhu, H., Suter, J. D., Sun, Y., 2008. Sensitive optical biosensors for unlabeled targets: A review. analytica chimica acta $620(1), 8-26$.

9 Fard, S. T., Donzella, V., Schmidt, S. A., Flueckiger, J., Grist, S. M., Talebi Fard, P., Wu, Y., Bojko, R. J., Kwok, E., Jaeger, N. A., et al., 2014. Performance of ultra-thin soi-based resonators for sensing applications. Optics Express 22 (12), 14166-14179.

10 Ghasemi, F., Chamanzar, M., Eftekhar, A. A., Adibi, A., 2014. An efficient technique for the reduction of wavelength noise in 360 resonance-based integrated photonic sensors. Analyst 139 (22), $5902-5911$.

11 Ghasemi, F., Eftekhar, A. A., Gottfried, D. S., Song, X., Cummings, R. D., Adibi, A., 2013. Self-referenced silicon nitride array microring biosensor for toxin detection using glycans at visible wavelength. In: SPIE BiOS. International Society for Optics and Photonics, pp. 85940A-85940A.

12 Ghasemi, F., Zhou, H., Adibi, A., 2015. Closed-form relations for resonance detection error using statistical analysis of amplitude noise. IEEE JOURNAL OF SELECTED TOPICS IN QUANTUM ELECTRONICS 21 (4), 9000308.

13 Gülşen, G., Naci Inci, M., 2002. Thermal optical properties of tio2 films. Optical materials 18 (4), 373-381.

14 Gylfason, K. B., Carlborg, C. F., Kazmierczak, A., Dortu, F., Vivien, L., Barrios, C. A., van der Wijngaart, W., Stemme, G., et al., 2010. On-chip temperature compensation in an integrated slot-waveguide ring resonator refractive index sensor array. Optics express 18 (4), 3226-3237.

3755 Heideman, R., Hoekman, M., Schreuder, E., 2012. Triplex-based integrated optical ring resonators for lab-on-a-chip and environmental detection. Selected Topics in Quantum Electronics, IEEE Journal of 18 (5), 1583-1596.

16 Hunt, H. K., Armani, A. M., 2010. Label-free biological and chemical sensors. Nanoscale 2 (9), 1544-1559.

17 Kirk, J. T., Fridley, G. E., Chamberlain, J. W., Christensen, E. D., Hochberg, M., Ratner, D. M., 2011. Multiplexed inkjet functionalization of silicon photonic biosensors. Lab on a Chip 11 (7), 1372-1377.

378 Lee, S., Eom, S. C., Chang, J. S., Huh, C., Sung, G. Y., Shin, J. H., 2010. Label-free optical biosensing using a horizontal air-slot sinx microdisk resonator. Optics express 18 (20), 20638-20644.

19 Luchansky, M. S., Washburn, A. L., Martin, T. A., Iqbal, M., Gunn, L. C., Bailey, R. C., 2010. Characterization of the evanescent field profile and bound mass sensitivity of a label-free silicon photonic microring resonator biosensing platform. Biosensors and Bioelectronics 26 (4), 1283-1291.

${ }_{320}$ Park, M. K., Kee, J. S., Quah, J. Y., Netto, V., Song, J., Fang, Q., La Fosse, E. M., Lo, G.-Q., 2013. Label-free aptamer sensor based on silicon microring resonators. Sensors and Actuators B: Chemical 176, 552-559.

21 Qavi, A. J., Bailey, R. C., 2010. Multiplexed detection and label-free quantitation of micrornas using arrays of silicon photonic microring resonators. Angewandte Chemie 122 (27), 4712-4715.

22 Quan, X., Fry, E. S., 1995. Empirical equation for the index of refraction of seawater. Applied Optics 34 (18), 3477-3480.

3233 Rong, G., Ryckman, J. D., Mernaugh, R. L., Weiss, S. M., 2008. Label-free porous silicon membrane waveguide for dna sensing. Applied Physics Letters 93 (16), 161109-161109.

24 Shah Hosseini, E., Yegnanarayanan, S., Atabaki, A. H., Soltani, M., Adibi, A., 2010. Systematic design and fabrication of 
high-q single-mode pulley-coupled planar silicon nitride microdisk resonators at visible wavelengths. Optics express 18 (3), 2127-2136.

3255 Smith, D. F., Song, X., Cummings, R. D., 2010. Chapter nineteen-use of glycan microarrays to explore specificity of glycanbinding proteins. Methods in enzymology 480, 417-444.

26 Soltani, M., Yegnanarayanan, S., Li, Q., Adibi, A., 2010. Systematic engineering of waveguide-resonator coupling for silicon microring/microdisk/racetrack resonators: theory and experiment. Quantum Electronics, IEEE Journal of 46 (8), 1158-1169.

27 Song, X., Lasanajak, Y., Xia, B., Heimburg-Molinaro, J., Rhea, J. M., Ju, H., Zhao, C., Molinaro, R. J., Cummings, R. D., Smith, 395 D. F., 2011. Shotgun glycomics: a microarray strategy for functional glycomics. Nature methods 8 (1), 85-90.

28 Song, X., Xia, B., Stowell, S. R., Lasanajak, Y., Smith, D. F., Cummings, R. D., 2009. Novel fluorescent glycan microarray strategy reveals ligands for galectins. Chemistry \& biology 16 (1), 36-47.

29 von Gunten, S., Smith, D. F., Cummings, R. D., Riedel, S., Miescher, S., Schaub, A., Hamilton, R. G., Bochner, B. S., Jun. 2009. Intravenous immunoglobulin contains a broad repertoire of anticarbohydrate antibodies that is not restricted to the IgG2 $400 \quad$ subclass. The Journal of allergy and clinical immunology 123 (6), 1268-76.e15.

30 Washburn, A. L., Luchansky, M. S., Bowman, A. L., Bailey, R. C., 2009. Quantitative, label-free detection of five protein biomarkers using multiplexed arrays of silicon photonic microring resonators. Analytical chemistry 82 (1), 69-72.

31 White, I. M., Fan, X., 2008. On the performance quantification of resonant refractive index sensors. Optics Express 16 (2), $1020-1028$.

4032 Xu, D., Densmore, A., Delâge, A., Waldron, P., McKinnon, R., Janz, S., Lapointe, J., Lopinski, G., Mischki, T., Post, E., et al., 2008. Folded cavity soi microring sensors for high sensitivity and real time measurement of biomolecular binding. Optics Express 16 (19), 15137-15148.

33 Zhu, H., White, I. M., Suter, J. D., Dale, P. S., Fan, X., 2007. Analysis of biomolecule detection with optofluidic ring resonator sensors. Optics Express 15 (15), 9139-9146. 